\section{Asociación entre obesidad y cáncer de ovario}

\author{
MACARENA VALLADARES PHD ${ }^{1}$, GINO CORSINI PHD ${ }^{1}$, \\ CARMEN ROMERO MSC ${ }^{2}$
}

\section{Association between obesity and ovarian cancer}

Obesity is a risk factor for cancer. Epidemiological evidences associate ovarian cancer with obesity. Epithelial ovarian cancer (EOC) is the most common type of ovarian cancer and accounts for a high rate of mortality. The association between ovarian cancer and obesity could be explained by molecular factors secreted by adipose tissue such as leptin. In EOC, leptin increases cell proliferation and inhibits apoptosis. Additionally, adipose tissue synthesizes endogenous estrogens, which increase cell proliferation of epithelial ovarian cells. Also, obesity associated hyperinsulinism could increase ovarian estrogen secretion.

(Rev Med Chile 2014; 142: 593-598)

Key words: Leptin; Obesity; Ovarian neoplasms.
${ }^{1}$ Centro de Investigación Biomédica (CIB), Facultad de Medicina, Universidad Diego Portales.

2Laboratorio de Endocrinología y Biología de la Reproducción. Hospital Clínico Universidad de Chile.

Recibido el 30 de octubre de 2012, aceptado el 29 de octubre de 2013.

\section{Correspondencia a:}

Dra. Macarena Valladares Centro de Investigación Biomédica (CIB), Facultad de Medicina Universidad Diego Portales.

Av. Ejército 141, Santiago, Chile. macarena.valladaresv@mail. udp.cl
L a obesidad es una enfermedad multifactorial producida por la interacción de factores genéticos y ambientales causados por el estilo de vida. Se caracteriza por un aumento excesivo de la grasa corporal, producido por un desequilibrio en el control homeostático mantenido en el tiempo, entre la energía consumida en la dieta y el gasto energético total ${ }^{1-4}$.

En países desarrollados y en vías de desarrollo existe un aumento en la prevalencia de obesidad, tanto en la población infantil como adulta ${ }^{5,6}$. En Chile, también se observa un aumento de la obesidad, donde $25,1 \%$ de los adultos presenta obesidad (definido como índice de masa corporal IMC $\geq 30$ ) (encuesta nacional de salud MINSAL 2010. www.minsal.cl).

Debido a la condición fisiológica que se produce en la obesidad, esta constituye un factor de riesgo para diferentes patologías, entre las cuales se encuentran la diabetes tipo 2, hipertensión arterial, patologías cardiovasculares, y diferentes tipos de cáncer ${ }^{2,3,7}$. En este sentido, la obesidad ha sido fuertemente asociada con cánceres de origen ginecológico como el de mama y endometrio ${ }^{8-10}$, sin embargo, con el cáncer de ovario la asociación encontrada es menos clara, y existen diferentes estudios al respecto.

En un meta-análisis de mujeres pre menopáusicas se estableció un aumento de $16 \%$ en desarrollar cáncer de ovario en mujeres adultas con sobrepeso (IMC entre 25 y $29 \mathrm{Kg} / \mathrm{m}^{2}$ ) y el riesgo aumenta a $30 \%$ en mujeres obesas (IMC $\left.\geq 30 \mathrm{Kg} / \mathrm{m}^{2}\right)^{11}$. Por otro lado, un estudio prospectivo de cohorte establece que un IMC $\geq 30 \mathrm{Kg} /$ $\mathrm{m}^{2}$ tiene un riesgo relativo de 1,3 de desarrollar cáncer de ovario en mujeres pre-menopáusicas; sin embargo, dicha asociación no se observa en mujeres post-menopáusicas ${ }^{12}$. En concordancia con esto, un estudio poblacional muestra que las mujeres obesas que no utilizan terapia de reemplazo hormonal tienen un riesgo mayor $(80 \%)$ de desarrollar cáncer de ovario respecto a las mujeres de peso normal ${ }^{13}$. Finalmente, lo anterior se relaciona con un meta-análisis reciente donde se encuentra que las personas no obesas tendrían una mejor sobrevida al cáncer de ovario versus las obesas y que esta asociación podría relacionarse con las características histológicas del cáncer ${ }^{14,15}$.

La presente revisión tiene como objetivo presentar los antecedentes que específicamente 
asocian a la obesidad con el cáncer de ovario, centrados principalmente en las señales moleculares que secreta el tejido adiposo y que contribuirían al desarrollo de este cáncer. Primero se expondrán las características generales del cáncer de ovario y del tejido adiposo. Luego, se discutirán las señales moleculares que genera el tejido adiposo y que permiten específicamente asociarlo con el desarrollo del cáncer de ovario.

\section{Cáncer de ovario}

El cáncer de ovario constituye la cuarta causa de muerte por cáncer en la población occidental, siendo el cáncer de ovario epitelial (COE) el más habitual dentro de ellos (80\%-90\%), además constituye la patología ginecológica que presenta mayor letalidad. Lo anterior se debe principalmente a la escasa sintomatología asociada, lo que produce una detección tardía de esta enfermedad ${ }^{16-18}$.

Se han definido diferentes factores que han sido asociados con un alto riesgo de desarrollar cáncer de ovario, tales como: terapia de reemplazo hormonal, historia familiar e infertilidad ${ }^{19}$. Sin embargo, existe una hipótesis propuesta por Risch y cols. que ha sido considerada como una causa importante para el desarrollo del cáncer de ovario llamada "ovulación incesante", la cual propone que durante cada ovulación el epitelio del ovario experimenta alteraciones. Debido a esto, hay una renovación constante del tejido epitelial del ovario y, por lo tanto, una alta estimulación con gonadotrofinas y estrógenos, lo que condicionaría a la malignización celular ${ }^{20}$. Por otro lado, existen diferentes factores que han sido asociados con un bajo riesgo de desarrollar cáncer de ovario, dentro de ellos se encuentran: el uso de anticonceptivos orales, haber tenido uno o más embarazos, ligaduras de trompas e histerectomía ${ }^{21,22}$.

Dentro de las principales características del cáncer de ovario se encuentra una alta angiogénesis (formación de nuevos vasos sanguíneos) regulada principalmente por el factor de crecimiento de endotelio vascular (VEGF), por el factor de crecimiento nervioso (NGF) y por su receptor específico del tipo tirosina/quinasa $\mathrm{TRKA}^{22,23}$. El proceso de angiogénesis se refleja en la alta expresión de VEGF (estimulada en parte por NGF), y de su transcrito en $\mathrm{COE}$ respecto a ovarios norma$\operatorname{les}^{24-26}$. Lo anterior contribuiría al alto crecimiento, y agresividad (metástasis) del cáncer de ovario lo que se traduce en una baja tasa de sobrevida ${ }^{27}$.

Dentro de los procesos cancerígenos que se producen en el COE, y en general en todos los tipos de cáncer, es que se genera una desregulación de la apoptosis y de la proliferación celular. La apoptosis o muerte celular programada es un proceso activo, altamente regulado y controlado genéticamente, mediante el cual células disfuncionales son eliminadas manteniendo así la homeostasis tisular y por ende, la función normal de los tejidos. Existen factores extracelulares o intracelulares que activan la apoptosis $28-30$. Por otro lado, la proliferación celular es el incremento del número de células por división celular ${ }^{31}$. De esta manera cuando se gatillan los procesos cancerígenos en el ovario se generan las señales moleculares dentro de la célula para que se estimulen los procesos de proliferación celular y se inhiban los mecanismos de la apoptosis, lo que permite finalmente que las células se dividan en un proceso absolutamente desregulado ${ }^{32}$.

\section{Tejido adiposo y su asociación con cáncer de ovario}

El tejido adiposo es un tejido endocrino que acumula la mayor cantidad de reserva energética en el organismo. Está compuesto de diferentes tipos de células (adipocitos, preadipocitos, fibroblastos, células endoteliales y macrófagos) las cuales sintetizan diferentes tipos de moléculas tales como: citoquinas, factores de crecimiento y adipoquinas. Estas últimas constituyen las principales moléculas secretadas por el tejido adiposo y son principalmente la leptina y adiponectina ${ }^{33-35}$. Además, los adipocitos secretan VEGF y estrógenos, los cuales contribuyen al desarrollo de la angiogénesis y proliferación celular respectivamente en el cáncer de ovario ${ }^{26,36}$.

La función primaria de la leptina es regular la ingesta de alimentos y el consumo de energía y sus niveles circulantes son directamente proporcionales con la cantidad de tejido graso ${ }^{37}$. La leptina realiza su acción a través de la unión a su receptor de transmembrana específico (LEPR), el cual se expresa principalmente a nivel del hipotálamo, sin embargo, también se ha descrito su expresión en tejidos periféricos como el ovario ${ }^{38,39}$. La cascada de señales que activa LEPR corresponde a 
las proteínas JAK2/STAT3; no obstante, también puede activar la vía Ras/ERK1/2 y PI-3K/Akt/ GSK3 que se ha descrito que regulan procesos de proliferación celular y apoptosis en el ovario. Esto muestra que la hormona leptina secretada por el tejido adiposo puede actuar a nivel ovárico y además regular procesos claves de la célula como la apoptosis y la proliferación celular, lo que constituye una relación directa entre obesidad y cáncer de ovario ${ }^{40,41}$.

En estudios in vitro se ha descrito que en líneas celulares de COE donde se sobrexpresa LEPR y este se activa, aumenta la proliferación celular a través de la vía ERK 1/2 y STAT-3 y dicho efecto se bloquea en presencia de inhibidores de ambas vías, lo que demuestra la participación específica de dicha cascada en procesos celulares a nivel ovárico ${ }^{42}$. Adicionalmente, este estudio establece que la leptina produce inhibición de la apoptosis, a través de la activación de las proteínas quinasas activadas por mitógenos (MAPK) en líneas celulares de cáncer de ovario (Figura 1). Por otro lado, se estableció que la leptina activa los receptores de estrógenos aumentando la transcripción del gen, en líneas celulares de cáncer de ovario, lo que produce un aumento de la proliferación celular. Además, los autores descartan que la leptina produzca un aumento de estrógenos directamente ${ }^{43}$. De esta manera, estos antecedentes muestran que una hormona secretada por el tejido adiposo, la lepti- na, genera dos efectos en el ovario: aumento de la proliferación celular e inhibición de la apoptosis, cuya cascada de señalización ha sido descrita, lo que contribuiría al desarrollo del cáncer de ovario $\mathrm{y}$, por lo tanto, permite asociar a nivel molecular la obesidad y el desarrollo del cáncer de ovario ${ }^{44}$.

En relación con la adiponectina que secreta el tejido adiposo en mayor cantidad y que en obesos se encuentra disminuida, existen antecedentes que la relacionan con procesos moleculares de desarrollo de otros cánceres ginecológicos como el cáncer de mama y endometrio ${ }^{45-47}$. Sin embargo, no existen antecedentes que la asocien específicamente con el cáncer de ovario. No obstante, se ha asociado la relación entre los niveles plasmáticos de leptina/adiponectina (L: A) con cáncer de ovario. De esta manera una baja relación L: A muestra un mayor tiempo de sobrevida (57 meses) en pacientes con COE, respecto a relaciones medias o altas (48 y 37 meses respectivamente). Sin embargo, en un análisis multivariado dicha asociación no se observa y sólo se mantiene la asociación del cáncer de ovario con la edad y el IMC y, por lo tanto, es necesario investigar más la relación de la adiponectina y procesos cancerígenos en el ovario $^{49}$. Por lo anterior es importante realizar estudios que puedan determinar algún efecto de la adiponectina directamente sobre el ovario en la regulación de la proliferación o apoptosis celular, como se ha observado en otros tipos de cáncer.

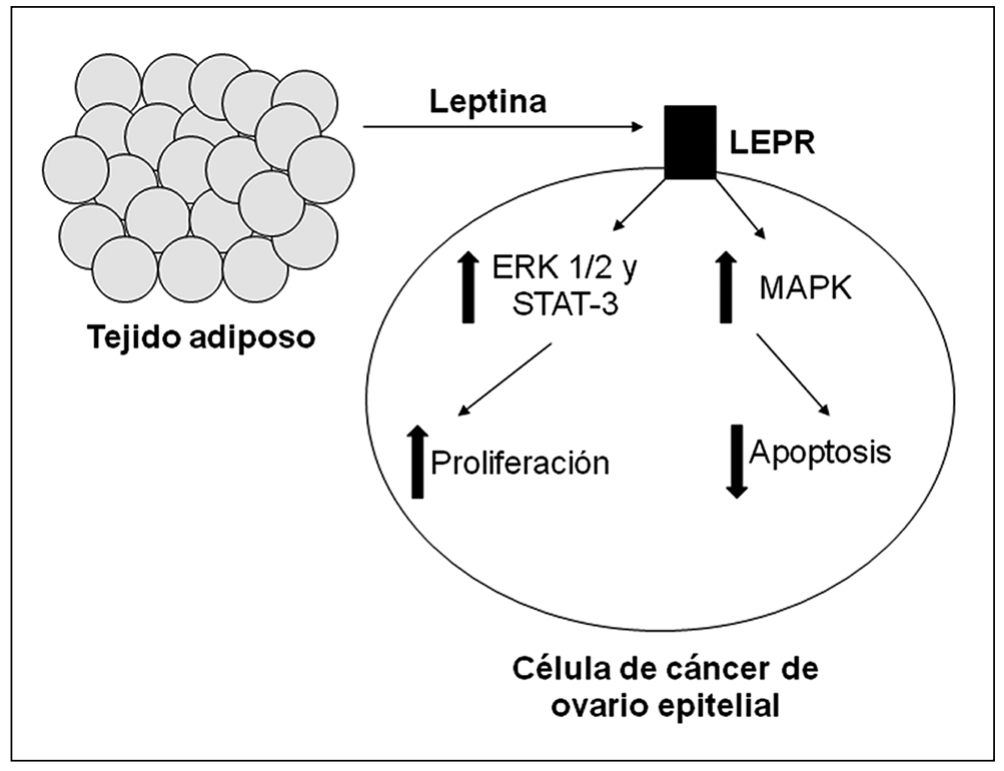

Figura 1. Acción de la leptina sobre células de cáncer de ovario epitelial. Se observa la acción de la leptina (secretada por el tejido adiposo) sobre células de cáncer de ovario epitelial, donde produce aumento de la proliferación, a través, de la activación de ERK $1 / 2$ y STAT-3 y disminución de la apoptosis mediante la activación de MAPK. LEPR: Receptor de leptina, ERK: Quinasa regulada por señales extracelulares, MAPK: Quinasa activada por mitógeno. 
Por otro lado, en el tejido adiposo también se produce síntesis de estrógenos endógenos debido a la aromatización de andrógenos. Lo anterior es otro proceso que permite asociar la obesidad con el cáncer de ovario, ya que los estrógenos producidos provocan un aumento en la proliferación celular de la superficie epitelial del ovario, lo cual puede contribuir al desarrollo del cáncer de ovario, en aquellos subgrupo de pacientes $\mathrm{COE}$ que expresan el receptor a estrógenos ${ }^{48}$. En relación a esto, el efecto de la terapia de reemplazo hormonal con el desarrollo del cáncer de ovario no está claramente determinado. Si bien dicha terapia ha sido asociada generalmente como un factor de riesgo del cáncer de ovario ${ }^{19}$, un meta-análisis establece que no observaron diferencias entre IMC y cáncer de ovario entre mujeres post-menopáusicas que utilizaron terapia de reemplazo hormonal y las que no. Este estudio, además, establece que la obesidad aumenta el riesgo de desarrollar los cáncer de ovario histológicamente menos frecuentes (serosos que presentan un alto grado de agresividad y los borderline) $\mathrm{y}$, finalmente, los autores concluyen que un bajo IMC no previene la letalidad del cáncer de ovario, por lo tanto, no actuaría como un factor protector ${ }^{15}$.

Dentro de la condición obesogénica se produce además una hiperinsulinemia, lo que provoca un aumento en los niveles circulantes del factor de crecimiento similar de insulina (IGF I) que generaría un aumento, por parte del ovario, en la conversión de andrógenos a estrógenos $\mathrm{y}$, como se ha establecido, los estrógenos endógenos aumentan la proliferación de células epiteliales del ovario, lo que aumenta el riesgo de que ocurran mutaciones celulares ${ }^{48,20}$.

De acuerdo a estos últimos antecedentes expuestos, la obesidad también puede ser asociada con el desarrollo del cáncer de ovario a través de la producción endógena de estrógenos y que al igual que la leptina produce alteraciones en los procesos celulares de apoptosis y proliferación celular directamente en el ovario.

\section{Conclusión}

Mediante esta revisión es posible establecer que tanto la obesidad como el cáncer de ovario dependen de una serie de factores distintos, esta situación muchas veces dificulta el poder encontrar una asociación clara entre ambas patologías.
Con el objetivo de establecer una asociación entre obesidad y cáncer de ovario, primero se revisaron los estudios epidemiológicos y luego los estudios moleculares que pueden explicar lo observado en los estudios poblacionales.

En relación a las señales moleculares generadas en el tejido adiposo y asociadas con el desarrollo del cáncer de ovario, el efecto de la leptina ha sido asociado con COE, sin embargo, no existen antecedentes del efecto de otras adipoquinas en la proliferación y apoptosis de células del ovario. Además, el efecto de los estrógenos endógenos producidos en el tejido adiposo también ha sido relacionado con el desarrollo del COE en cierto grupo de pacientes.

Por otro lado, el efecto de la adiponectina ha sido menos estudiado y sólo se ha establecido que tendría asociación con el cáncer de ovario debido a la relación entre los niveles plasmáticos de leptina/ adiponectina. Podría constituir un foco de estudio a futuro considerando que es la adipoquina que más secreta el tejido adiposo y su efecto se ha establecido en otros cánceres ginecológicos.

De acuerdo a lo expuesto en esta revisión, queda de manifiesto que debido a los altos índices de obesidad de nuestra población y a la alta letalidad que presenta el cáncer de ovario, la relación entre ambas enfermedades constituye un campo importante de estudio. Lo anterior se orienta a poder generar políticas públicas que eduquen sobre los riesgos de desarrollar obesidad asociados al cáncer de ovario, considerando, además, que la obesidad constituye un factor de riesgo modificable para el cáncer de ovario. Asimismo, y lo que podría constituir lo más novedoso en esta área de estudio, es poder generar terapias para el tratamiento del cáncer de ovario. Esto último se podría orientar a actuar sobre las cascadas de señales, que han sido descritas en esta revisión, que activa la leptina y que al hacerlo regula los procesos de proliferación celular y apoptosis en el ovario.

Además de la leptina, la asociación entre obesidad y cáncer de ovario también se gatillaría por la producción de estrógenos endógenos en el tejido adiposo, condición que también se ve aumentada en una condición obesogénica. En este caso, las pacientes afectadas por esta situación del aumento de estrógenos endógenos serían exclusivamente aquellas que expresan el receptor de estrógenos en el cáncer de ovario. Considerando el efecto de los estrógenos endógenos y que la leptina puede 
activar los receptores de estrógenos en células de cáncer de ovario, el papel de los receptores de estrógenos como un factor del pronóstico del cáncer de ovario aún no está claramente definido como sí lo es en el cáncer de mama. Lo que ha sido definido es que las pacientes que presenten obesidad y expresen en el cáncer de ovario receptores de estrógenos podrían presentar un mayor riesgo de desarrollar cáncer de ovario.

Finalmente, se puede establecer que, de acuerdo a los antecedentes expuestos, existe una asociación entre obesidad y cáncer de ovario, sin embargo, las asociaciones encontradas son moderadas lo que se puede deber a que el cáncer de ovario depende de diferentes factores, lo cual dificulta determinar una causalidad entre obesidad y cáncer de ovario. No obstante, son necesarios estudios a futuro para determinar otras moléculas secretadas por el tejido adiposo o generadas a partir de la condición obesogénica que pueden provocar el desarrollo del cáncer de ovario.

\section{Referencias}

1. Matheson EM, King DE, Everett CJ. Healthy lifestyle habits and mortality in overweight and obese individuals. J Am Board Fam Med 2012; 25 (1): 9-15.

2. Jensen MK, Chiuve SE, Rimm EB, Dethlefsen C, Tjønneland A, Joensen AM, et al. Obesity, behavioral lifestyle factors, and risk of acute coronary events. Circulation 2008; 17 (24): 3062-9.

3. Peeters A, Barendregt JJ, Willekens F, Mackenbach JP, Al Mamun A, Bonneux L. Obesity in adulthood and its consequences for life expectancy: a life-table analysis; NEDCOM, the Netherlands Epidemiology and Demography Compression of Morbidity Research Group. Ann Intern Med 2003; 138 (1): 24-32.

4. Farooqi IS. Genetic, molecular and physiological insights into human obesity. Eur J Clin Invest 2011; 41 (4): 4515.

5. Hossain P, Kawar B, El Nahas M. Obesity and diabetes in the developing world-a growing challenge. $\mathrm{N}$ Engl J Med 2007; 356 (3): 213-5.

6. Egger G, Swinburn B, Islam FM. Economic growth and obesity: an interesting relationship with world-wide implications. Econ Hum Biol 2012; 10 (2): 147-53.

7. Fair AM, Montgomery K. Energy balance, physical activity, and cancer risk. Methods Mol Biol 2009; 472: 57-88.

8. Aguilar Cordero MJ, Neri Sánchez M, Padilla López CA, Pimentel Ramírez ML, García Rillo A, Mur Villar
N. Overweight/obesity in women and its implication in breast cancer: age of diagnosis. Nutr Hosp 2012; 27 (5): 1643-7.

9. Amadou A, Hainaut P, Romieu I. Role of obesity in the risk of breast cancer: lessons from anthropometry. J Oncol 2013 (en prensa).

10. Goodman MT, Hankin JH, Wilkens LR, Lyu LC, McDuffie K, Liu LQ, et al. Diet, body size, physical activity, and the risk of endometrial cancer. Cancer Res 1997; 15 (22): 5077-85.

11. Olsen CM, Green AC, Whiteman DC, Sadeghi S, Kolahdooz F, Webb PM. Obesity and the risk of epithelial ovarian cancer: a systematic review and meta-analysis. Eur J Cancer 2007; 43 (4): 690-709.

12. Schouten LJ, Rivera C, Hunter DJ, Spiegelman D, Adami HO, Arslan A. Height, body mass index, and ovarian cancer: a pooled analysis of 12 cohort studies. Cancer Epidemiol Biomarkers Prev 2008; 17 (4): 902-12.

13. Leitzmann MF, Koebnick C, Danforth KN, Brinton LA, Moore SC, Hollenbeck, et al. Body mass index and risk of ovarian cancer. Cancer 2009; 115: 812-22.

14. Olsen CM, Nagle CM, Whiteman DC, Ness R, Pearce $\mathrm{CL}$, Pike MC, et al. Obesity and risk of ovarian cancer subtypes: evidence from the Ovarian Cancer Association Consortium. Endocr Relat Cancer 2013; 22 (2): 251-62.

15. Protani MM, Nagle CM, Webb PM. Obesity and ovarian cancer survival: a systematic review and meta-analysis. Cancer Prev Res (Phila) 2012; 5 (7): 901-10.

16. Ali AY, Farrand L, Kim JY, Byun S, Suh JY, Lee HJ, et al. Molecular determinants of ovarian cancer chemoresistance: new insights into an old conundrum. Ann N Y Acad Sci 2012; 1271: 58-67.

17. Jemal A, Siegel R, Ward E, Hao Y, Xu J, Murray T, et al. Cancer statistics, 2008. CA Cancer J Clin 2008; 58 (2): 71-96.

18. Landen CN Jr, Birrer MJ, Sood AK. Early events in the pathogenesis of epithelial ovarian cancer. J Clin Oncol 2008; 26 (6): 995-1005.

19. Leitzmann MF, Koebnick C, Danforth KN, Brinton LA, Moore SC, Hollenbeck, et al. Body mass index and risk of ovarian cancer. Cancer 2009; 115: 812-22.

20. Risch HA. Hormonal etiology of epithelial ovarian cancer, with a hypothesis concerning the role of androgens and progesterone. J Natl Cancer Inst 1998; 90: 1774-86.

21. Beral V, Doll R, Hermon C, Peto R, Reeves G. Ovarian cancer and oral contraceptives: collaborative reanalysis of data from 45 epidemiological studies including 23,257 women with ovarian cancer and 87,303 controls. Lancet 2008; 371: 303-14.

22. Lukanova A, Kaaks R. Endogenous hormones and ovarian cancer: epidemiology and current hypotheses. 
Cancer Epidemiol Biomarkers Prev 2005; 14: 98-107.

23. Tapia V, Gabler F, Muñoz M, Yazigi R, Paredes A, Selman A, et al. Tyrosine kinase A receptor (trkA): a potential marker in epithelial ovarian cancer. Gynecol Oncol 2011; 12: 13-23.

24. Julio-Pieper M, Lara H, Bravo J, Romero C. Effects of nerve growth factor (NGF) on blood vessels area and expression of the angiogenic factors VEGF and TGFbeta I in the rat ovary. Reprod Biol Endocrinol 2006; 4: 57-67.

25. Julio-Pieper M, Lozada P, Tapia V, Vega M, Miranda C, Vantman D, et al. Nerve growth factor induces vascular endotelial growth factor expression in granulose cells via a trkA receptor/mitogen-activated protein kinaseextracellularly regulated kinase 2-dependent pathway. J Clin Endocrinol Metab 2009; 94 (8): 3065-71.

26. Campos X, Muñoz Y, Selman A, Yazigi R, Moyano L, Weinstein-Oppenheimer C, et al. Nerve growth factor and its high-affinity receptor TrkA participate in the control of vascular endothelial growth factor expression in epithelial ovarian cancer. Gynecol Oncol 2007; 104: 168-75.

27. Neufeld G, Cohen T, Gengrinivich S, Poltorak Z. Vascular endothelial growth factor (VEGF) and its receptors. FASEB J 1999; 13 (11): 9-22.

28. Elmore, S. Apoptosis: A Review of Programmed Cell Death. Toxicologic Pathology 2007; 35: 495-516.

29. Du C, Fang M, Li Y, Li L, Wang X. Smac, a mitochondrial protein that promotes cytochrome c-dependent caspase activation by eliminating IAP inhibition. Cell 2000; 102: 33-42.

30. Locksley RM, Killeen N, Lenardo MJ. The TNF and TNF receptor superfamilies: integrating mammalian biology. Cell 2001; 104: 487-501.

31. Holland-Frei. Cancer medicine. En: C Bast, Jr, Donald W Kufe, Raphael E Pollock, Ralph R, et al. Cell Proliferation, Differentiation, and Apoptosis. Atlanta, USA: editorial Decker Ltda. 2003; p. 95-115.

32. Johnstone RW, Ruefli AA, Lowe SW. Apoptosis: a link between cancer genetics and chemotherapy. Cell 2002; 108: 153-64.

33. Van Kruijsdijk R, van der Wall E, Visseren F. Obesity and cancer: the role of dysfunctional adipose tissue. Cancer Epidemiol Biomarkers Prev 2009; 18 (10): 256978.

34. Redinger RN. The physiology of adiposity. J Ky Med Assoc 2008; 106: 53-62.

35. Guilherme A, Virbasis JV, Puri V, Czech MP. Adipocyte dysfunctions linking obesity to insulin resistance and type 2 diabetes. Mol Cell Biol 2008; 9 (5): 367-77.
36. Syed V, Ulinski G, Mok SC, Yiu GK, Ho SM. Expression of gonadotropin receptor and growth responses to key reproductive hormones in normal and malignant human ovarian surface epithelial cells. Cancer Res 2001; 61: 6768-76.

37. Anderlova K, Kremen J, Dolezalova R, Housova J, Haluzikova D, Kunesova M, et al. The influence of verylow-calorie-diet on serum leptin, soluble leptin receptor, adiponectin and resistin levels in obese women. Physiol Res 2006; 55: 277-83.

38. Tartaglia LA. The leptin receptor. J Biol Chem 1997; 272 (10): 6093-6.

39. Mantzos F, Vanakara P, Samara S, Wozniak G, Kollia P, Messinis I, et al. Leptin receptor expression in neoplastic and normal ovarian and endometrial tissue. Eur J Gynaecol Oncol 2011; 32 (1): 84-6.

40. Sweeney G. Leptin signalling. Cell Signal 2002; 14 (8): 655-63.

41. Zabeau L, Lavens D, Peelman F, Eyckerman S, Vandekerckhove J, et al. The ins and outs of leptin receptor activation. FEBS Lett 2003; 546 (1): 45-50.

42. Choi JH, Park SH, Leung PC, Choi KC. Expression of leptin receptors and potential effects of leptin on the cell growth and activation of mitogen-activated protein kinases in ovarian cancer cells. J Clin Endocrinol Metab 2005; 90 (1): 207-10.

43. Choi JH, Lee KT, Leung PC. Estrogen receptor alpha pathway is involved in leptin-induced ovarian cancer cell growth. Carcinogenesis 2011; 32: 589-96.

44. Uddin S, Bu R, Ahmed M, Abubaker J, Al-Dayel F, Bavi P. Overexpression of leptin receptor predicts an unfavorable outcome in Middle Eastern ovarian cancer. Mol Cancer 2009; 18: 1-12.

45. Mantzoros C, Petridou E, Dessypris N, Chavelas C, Dalamaga M, Alexe DM, et al. Adiponectin and breast cancer risk. J Clin Endocrinol Metab 2004; 89: 1102-7.

46. Miyoshi Y, Funahashi T, Kihara S, Taguchi T, Tamaki $\mathrm{Y}$, Matsuzawa $\mathrm{Y}$, et al. Association of serum adiponectin levels with breast cancer risk. Clin Cancer Res 2003; 9: 5699-704.

47. Cust AE, Kaaks R, Friedenreich. Plasma adiponectin levels and endometrial cancer risk in pre- and postmenopausal women. J Clin Endocrinol Metab 2007; 92: 255-63.

48. Díaz ES, Karlan BY, Li AJ. Obesity-associated adipokines correlate with survival in epithelial ovarian cancer. Gynecol Oncol 2013; 129 (2): 353-7.

49. Cunat S, Hoffmann P, Pujol P. Estrogens and epithelial ovarian cancer. Gynecol Oncol 2004; 94 (1): 25-32. 